\title{
Read-out electronics for a micromachined thermal conductivity and diffusivity sensor
}

\author{
Bernhard Mayrhofer ${ }^{1}$, Jochen Kuntner ${ }^{2}$, and Bernhard Jakoby ${ }^{1}$ \\ ${ }^{1}$ Institute for Microelectronics and Microsensors, Johannes Kepler University Linz, Altenberger Str. 69, \\ A-4040 Linz, Austria \\ ${ }^{2}$ Institute of Sensor and Actuator Systems; Vienna University of Technology, Gusshausstr. 27-29/366, \\ A-1040 Vienna, Austria ${ }^{1}$
}

\section{Motivation}

There are several classical methods for measuring the thermal conductivity of liquids. One of them is the hot wire method, where a heating wire,is embedded in the liquid being sensed [1]. A temperature element beside the heater is used to detect the heat emitted by the heater. Alternatively, the heating wire itself may be used as a temperature sensor. In the conventional hot wire method, the heater is powered by a DC source and the transient response at the sensor is used to obtain the thermal conductivity of the liquid (or gas).

In our approach, the hot wire method is modified. Firstly, a micromachined sensor element is employed and secondly, a sinusoidal signal is used to power the heater. The sensor thus measures the diffusive heat wave emitted by the heater where the phase and amplitude of the AC-component of the detected signal can be used to determine the thermal conductivity and diffusivity (and thus also the specific heat capacity) of the tested liquid [2]. In this paper we discuss suitable read-out electronics, which have been custom-built around the micromachined sensor, where the latter has been fabricated at the Institute of Sensor and Actuator Systems of the Vienna University of Technology. The previously required amount of measurement equipment facilitating the supply and readout of the sensor [2] was reduced to a single printed circuit board and a PC connected to the board via an USB interface.

\section{Sensor design and technology}

In the following we briefly review the technology associated with the sensor device, for details please refer to [1]. In order to fabricate a sensor utilizing the hot wire method, it is essential to provide an effective embedding of the heater in the material to be sensed and to avoid spurious thermal shunts as, e.g., represented by a substrate carrying the heater. For heaters realized in thin film technology on some carrier (substrate) it is thus very important to reduce the associated thermal masses and the spurious heat diffusion into the substrate. To reduce these effects, the structure of the heater and the thermal sensors can be realized on thin membranes or micro-bridges. The used sensor is based on this concept, where the entire chip features a size of $2 \mathrm{~mm} \times 4 \mathrm{~mm}$. It consists of a thin silicon nitride membrane supported by a $0.3 \mathrm{~mm}$ silicon frame. A chromium heater $(\mathrm{H})$ is located in the centre of this membrane (see Fig. 1). Beside the heater there are two highly sensitive amorphous germanium thermistors (MT1 and MT2). Amorphous germanium exhibits high values of both, resistivity and associated temperature coefficient. The specific resistivity is about $5 \Omega \mathrm{m}$ and the temperature coefficient of resistance is approximately $-2 \% / \mathrm{K}$ at room temperature. In order to measure the ambient temperature there are two further thermistors (ST1 and ST2) on the silicon frame of the membrane. The mounting of the sensor chip on a PCB-holder is shown in Fig. 2.

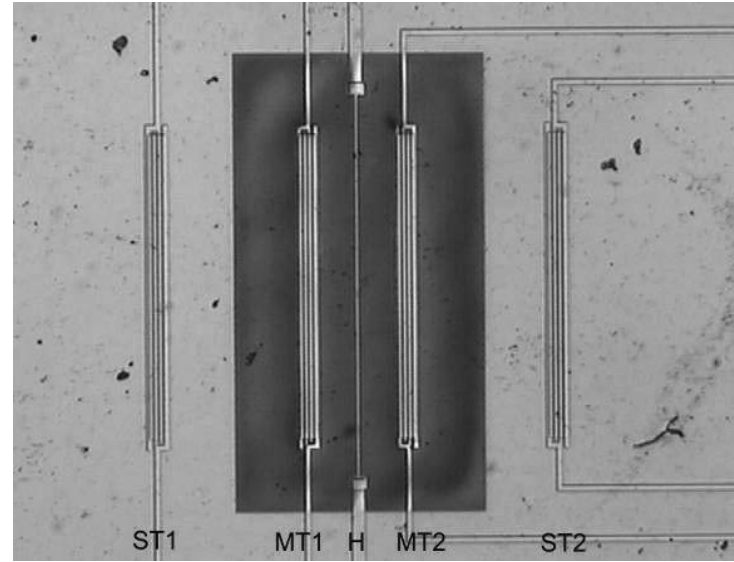

Fig. 1: Sensor detail: The dark area represents the membrane with the heater $\mathrm{H}$ and the termistors MT1 and MT2 next to it. Thermistors ST1 and ST2 are located on the silicon frame.

\footnotetext{
${ }^{1}$ Jochen Kunter is now with the Hilti Corporation, Schaan, Liechtenstein
} 


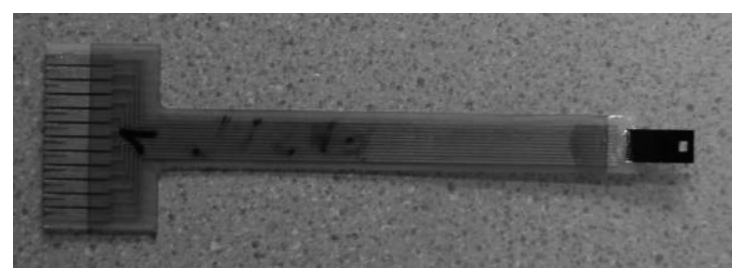

Fig. 2: Mounted sensor: The sensor chip is glued and bonded to a PCB-holder enabling measurements with the immersed chip.

\section{Measurement principle}

In order to measure the thermal conductivity $\lambda$ and diffusivity $a$ of the tested liquid, the sensor is immersed into the sample liquid, so the membrane is in intimate contact with the liquid on both sides. The thermal diffusivity is related to the specific heat capacity $c_{p}$ as follows:

$a=\frac{\lambda}{\rho \cdot c_{p}}$

where $\rho$ denotes the density. Now a sinusoidal voltage is applied to the heater so that a diffusive heat wave propagates from the heater $(\mathrm{H})$ into the surrounding liquid. The associated Joule heating $p_{\text {heater }}(t)$ is proportional to the square of the applied heater voltage such that it represents a sinussodial signal $p_{\text {heater, } A C}$ with frequency $\omega$ plus a DC-offset $p_{\text {heater, } D C}$. The associated frequency of the heater voltage is thus $\omega / 2$. The actually used frequencies are typically in the range below $10 \mathrm{~Hz}$. Thus for a heater voltage $u_{\text {heater }}(t)=\hat{U}_{\text {heater }} \cos (\omega t / 2)$ we have

$p_{\text {heater }}(t)=\frac{\left[u_{\text {heater }}(t)\right]^{2}}{R_{\text {heater }}}=\frac{\left[\hat{U}_{\text {heater }} \cdot \cos \left(\frac{\omega}{2} t\right)\right]^{2}}{R_{\text {heater }}}=\frac{\hat{U}_{\text {heater }}^{2} \cdot[1+\cos (\omega t)]}{2 \cdot R_{\text {heater }}}=\frac{\hat{U}_{\text {heater }}^{2}}{2 \cdot R_{\text {heater }}}+\underbrace{\frac{\hat{U}_{\text {heater }}^{2}}{2 \cdot R_{\text {heater }}} \cdot \cos (\omega t)}_{P_{\text {heater }, D C}}$.

For the fabricated prototypes, the heater's resistance at room temperature is $R_{\text {heater }}=2113 \Omega$ (the temperature coefficient of $R_{\text {heater }}$ is neglected in the above formula). The temperature response is measured by one of the membrane thermistors MT1 or MT2. An interface circuit described below provides a voltage corresponding to the excess temperature (with respect to ambient temperature) of the thermistor, which is composed of a DC component, representing the temperature increase associated with the DC-offset of the generated heat, superposed by an AC component $u_{\text {thermistor, } A C}(t)$ corresponding to the temperature variations induced by the AC component of the generated heat $p_{\text {heater, } A C}$ :

$$
u_{\text {thermistor }}(t)=U_{\text {thermistor }, D C}+\underbrace{\hat{U}_{\text {thermistor }} \cdot \cos (\omega t+\phi)}_{u_{\text {thermistor }, A C(t)}}
$$

The steady state amplitude $\hat{u}_{\text {thermistor, } A C}$ and phase $\phi$ of the AC component are influenced by the thermal properties of the liquid. Thus the thermal diffusivity and conductivity of a liquid can be determined by measuring the amplitude and the phase of the thermistor voltage $u_{\text {thermistor, } A C}(t)$.

In [1] a simple analytical model for the sensor was developed yielding a relation between the temperature variation and the heating power and frequency, the sensor geometry, thermal conductivity, and diffusivity of the tested liquid. By means of this model, if the thermal diffusivity $a$ and conductivity $\lambda$ of the measured liquid are known, the 
amplitude and phase of the excess temperature $\Delta T$ caused by the heater can be calculated (using complex notation) [1]:

$$
\Delta T(r, t)=\frac{\hat{P}_{\text {heater }}}{2 \cdot \pi \cdot \lambda}\left[\frac{1}{b} \int_{\Delta d+d}^{\Delta d+d+b} K_{0}\left(\sqrt{\frac{j \omega}{a} \cdot r}\right) d r+\frac{1}{b} \int_{d}^{d+b} K_{0}\left(\sqrt{\frac{j \omega}{a} \cdot r}\right) d r\right]
$$

Here $b, d$, and $\Delta d$ characterize the geometry of heater and thermistor in the cross-section of the device (for details see [1]). Since this relation contains the zeroth-order modified Bessel function $K_{0}$, it would be very difficult to invert the formula in order to obtain the liquid's thermal diffusivity and conductivity for a given (measured) excess temperature $\Delta T$. Alternatively, a nomogram is created by drawing characteristic curves for the amplitude and phase of $\Delta T$ as a function of different values of $a$ and $\lambda$. When a sample liquid is tested and $\Delta T$ (amplitude and phase) is measured, the thermal diffusivity and conductivity can be read out of the nomogram. The results of the measurement have errors because of inaccuracies in the analytical model of the sensor. These errors can be minimized by a calibration procedure described below involving three reference liquids.

\section{Read-out electronics}

The readout electronic consists of three parts:

- The sensor interface, which supplies the heater and detects the thermal response of the thermistors,

- a microcontroller that generates the sinusoidal signal for the heater voltage, samples that output voltage of the amplifier and sends the data to a host PC via USB, and

- a Labview ${ }^{\mathrm{TM}}$ program with an embedded Matlab ${ }^{\mathrm{TM}}$ script that communicates with the microcontroller, calculates and displays the thermal conductivity and diffusivity.

These functional blocks will be discussed below.

\subsection{The sensor interface}

The senor interface has two main tasks: the first one is to provide the sinusoidal voltage for the heater. The second task is the detection of changes of the membrane thermistor's resistance, which represent the thermal response $\Delta T$, and convert them into a voltage signal. This signal has to be in the range from 0 to $5 \mathrm{~V}$ fitting the input of the internal Analog-Digital-Converter of the utilized microcontroller PIC18F452.

The sinusoidal heater voltage is generated by a 8 bit Digital to Analog-Converter (DAC5573) which is connected to the microcontroller via an $I^{2} \mathrm{C}$-Bus. The data points for sine waveform are stored in a table located in the flash memory of the microcontroller. The DAC has an output range from $0 \mathrm{~V}$ to $2,5 \mathrm{~V}$ and so the output voltage is defined with a peak to peak value $V_{p p}=2,4 \mathrm{~V}$ and with an offset of $1,2 \mathrm{~V}$. Applying this voltage directly to the heater would cause harmonic and non linear components in the signal of the thermal response due to the latter DC-offset. So the output is connected to an amplifier stage subtracting this offset and at the same time providing a low output impedance driving the heater. VOUTA denotes the sinusoidal voltage generated by the AD-Converter and VOUTB is connected to another output channel of the ADC, which is adjusted to a level that reduces the offset of the heater voltage to zero.

In order to measure the temperature response, which is proportional to changes of membrane thermistor's resistance, one pin of this thermistor (MT1B) is connected to an other output of the DAC called VOUTC. By means of the developed microcontroller software, this output is by default set to fixed voltage level of $2 \mathrm{~V}$, but can be adjusted if necessary (e.g., to increase the sensitivity or to measure in environments with low thermal conductivities, etc.). The second pin of the membrane thermistor (MT1A) is connected to the input of the operational amplifier N2 (see Fig. 3), which is operated as a transimpedance amplifier.

The significant parameter for the measurement is the relative change of thermistor's resistance (corresponding to the excess temperature). However, the thermistor-resistance has a relatively large value of approximately $62 \mathrm{k}$ Ohms at room temperature, which causes a big offset in the amplifier's output signal, which would reduce the performance of the system in terms of sensitivity and resolution. This offset also cannot be simply minimized by subtracting a constant value, because it depends on the ambient temperature (and thus also on the temperature of the tested liquid), which may vary. Thus, the substrate thermistor ST1, which has the same resistance and temperature coefficient as MT1, is used to minimize this offset. Pin ST1A of the substrate thermistor (ST1) is connected to an inverting buffer amplifier N1 (Fig. 3), while the second pin (ST1B) is connected to VOUTC providing the measurement voltage for the membrane thermistor MT1. The inverted output signal of this amplifier is then added to the signal of MT1in terms of a current at the low-impedance input of the transimpedance amplifier N2. Hence the output signal of the amplifier N2 ideally corresponds to the excess temperature, only. A change in the ambient temperature or a general temperature drift caused by the liquid has the same influence on both thermistors MT1 and ST1; as they have the same temperature coefficient this change is compensated. The output of the amplifier N2 is connected to 
the amplifier N3 which amplifiers the signal by a gain of 5.1 , which, for the used sensor prototypes, yields reasonable signal amplitudes for common liquids. The output signal of this amplifier (N3) is sampled by the internal 10bit A/Dconverter of the microcontroller. The voltage divider consisting of R7 and R8 is connected to -5V and VOUTD (which can be controlled in the range 0 to $2.5 \mathrm{~V}$ by the software) feeding an additional input current to the compensation stage N2. This allows to correct slight inequalities in the nominal resistance value of the two thermistors MT1 and ST1 and further tolerances.

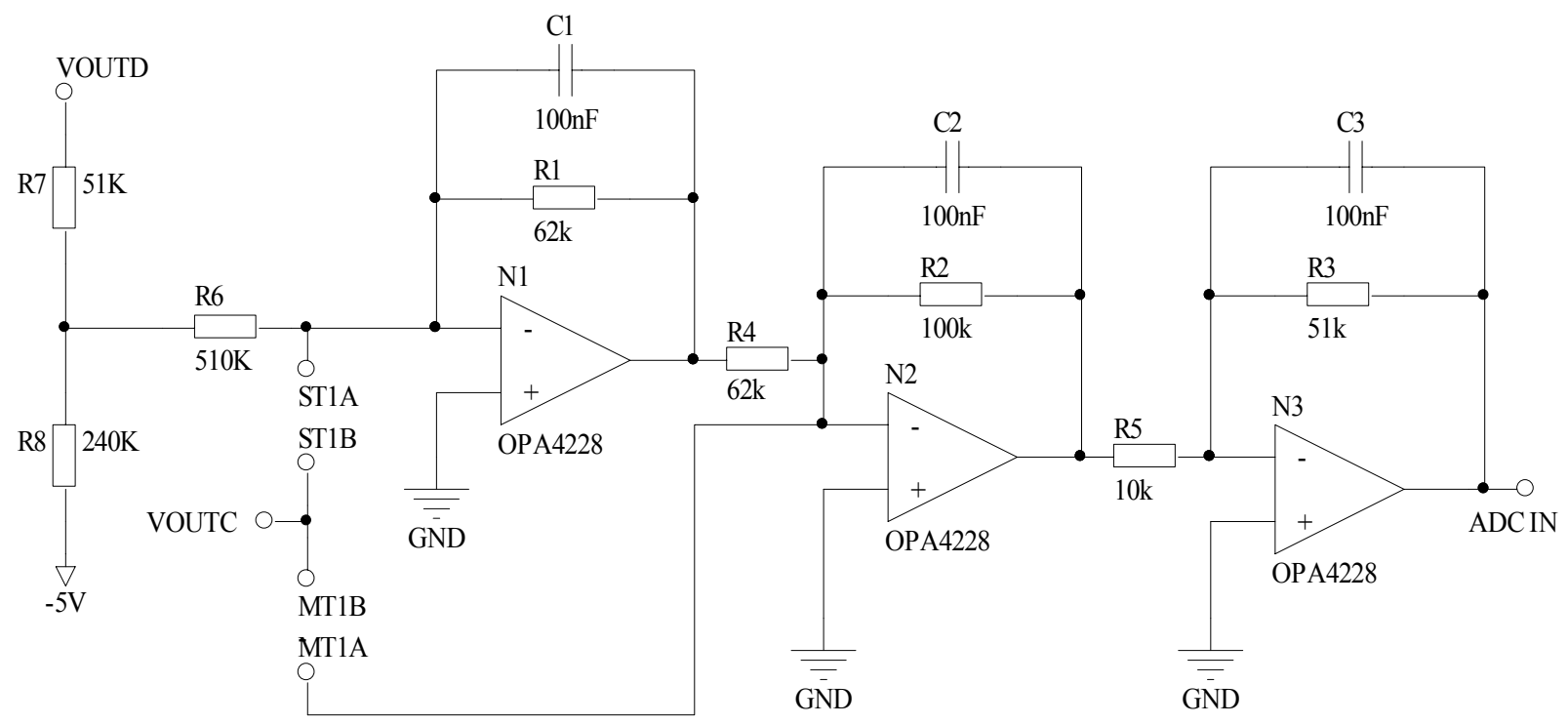

Fig. 3: Thermistor readout stage including offset cancellation.

\subsection{The microcontroller}

The microcontroller has three main tasks:

- $\quad$ Generating the cosine waveform data for the heater signal

- $\quad$ Sampling the output signal of the sensor interface

- $\quad$ Communication with the PC

The cosine curve is approximated by storing 100 equally spaced samples of one period of a cosine waveform in an array in flash memory of the microcontroller. To generate a cosine waveform at the microcontroller output, the $\mathrm{I}^{2} \mathrm{C}$ of the microcontroller is connected to a D/A-converter (DAC5573).

The measurement cycle and clocking of the cosine generation is implemented as follows. A timer of the controller is configured to count down from a specified value. If the value of the counter has reached zero, an interrupt is triggered and an interrupt service routine (ISR) starts. This first command in this ISR is reloading the timer value, such that the periodic triggering of the ISR is maintained in the sequel. Then one data point of the stored sine waveform is sent to the D/A-converter via I'C-bus. The ISR also utilizes a counter, counting the number of interrupts occurred, which is reset after 100 interrupts. Every trigger event another data point of the waveform is sent according to the value of the aforementioned counter. After 100 points, the procedure thus starts again with the first point. The result is a periodic sinusoidal voltage at the output of the digital to analog converter, with constant amplitude and offset, and a frequency depending on the starting value of the countdown-timer. In order to measure the exact phase between the input signal and the output signal of the sensor, it is important to have a time reference of the sample signal. To provide this, the sampling of the sensor output signal is also done in the same interrupt routine. Every time the D/A-converter is updated with the next data point, the internal $A / D$-converter is triggered to sample the current output sensor signal. The captured data, which is now exactly referenced to the generated waveform, is stored to an array in the memory of the microcontroller. Together with the captured sensor data, the associated value of reference sine signal and an associated cosine signal (obtained from another stored lookup table) is stored. Every time the measurement cycle is completed, the array containing the measurement data is copied to another array, which provides the most recent valid measurement data for transfer to the PC. The microcontroller is connected to the PC by a RS232 to USBConverter. Upon request, the sampled sensor signal and the reference signal are sent by the RS232-Port of the microcontroller and received at a Virtual-COM-Port on the PC. A LABVIEW-program on the computer controls the whole measurement procedure and processes the captured sensor data in order the display the thermal conductivity and diffusivity of the tested liquid (as described below). 


\subsection{The Labview program}

The Labview program triggers the microcontroller to start data transfer by sending a start command. The PC then receives three data arrays containing the most recent complete measurement data from the controller. The data arrays contain the following signals:

$s(i) \ldots$ sampled data of the sensor signal

$r s(i)$.. a reference sine signal

$r c(i) .$. a reference cosine signal

Note that the sine and cosine signals as well as the sampled sensor data show twice the frequency of the voltage applied to the heater (see section "Measurement Principle" above). To determine the thermal properties of the liquid it is necessary to know the magnitude and phase of the sampled signal with respect to the reference cosine signal. First we determine the in-phase $(x)$ and the quadrature component $(y)$ of the sensor signal $\left(i_{\max }\right.$ denotes the number of samples, in our case 100):

$$
\begin{gathered}
x=\sum_{i=0}^{i_{\max }-1} s(i) \cdot r c(i) \\
y=\sum_{i=0}^{i_{\max }-1} s(i) \cdot r s(i)
\end{gathered}
$$

where $i_{\max }$ is the size of the data array. The phase with respect to the cosine signal then is obtained as:

phase: $\varphi=-\operatorname{atan} 2(y, x)$,

where atan2 $(y, x)$ denotes the commonly used function that correctly computes the phase of a complex number $x+j y$ in a range $-\pi$ to $+\pi$ (whereas the arctangent of $y / x$ yields the correct phase angle in the range $-\pi / 2$ to $+\pi / 2$, only). A measure for the magnitude of the signal can be obtained as

magnitude: $m=\frac{2 \cdot \sqrt{x^{2}+y^{2}}}{i_{\max }}$.

In order to obtain $|\Delta T|$, the magnitude $m$ is multiplied by a conversion factor that takes account of the conversion gain of the $A D C$, the gain of the readout stage, and the temperature coefficient of the thermistor. Thus the phase and the amplitude of the complex valued $\Delta T$ are known and Eq. (5) could be used to obtain the thermal properties of the liquid. However, as this formula cannot be inverted easily to obtain the parameters $a$ and $\lambda$, i.e. the thermal diffusivity and conductivity, respectively, we use a simple fit procedure. As the phase of $\Delta T$ depends on $a$, only, we first vary the value of $a$ until the phase coincides with the measured phase. Then, keeping the so obtained value for $a$, the parameter $\lambda$ in the formula is varied until the magnitudes coincide as well.

The remaining errors, which are partly caused by inaccuracies of the analytical model being the foundation for the used formula (5), are minimized by a calibration procedure where the sensor is immersed in three sample liquids with known thermal properties. The analytical model is incorrect as in case of membrane-based devices there will be spurious thermal shunts through the membrane, which are not considered by (5). Furthermore (5) represents a $2 \mathrm{D}$ approximation to the actual 3D-problem. Finally errors in the conversion coefficient can be corrected using the calibration procedure. For the utilized simple calibration approach, a linear fit between nominal and measured values for the thermal conductivity and diffusivity of three reference liquids is employed. Further measurements (fit data) are then corrected by the slope and the offset resulting from the linear fit.

Fig. 4 and Fig. 5 show examples of this calibration for a particular device where water, ethanol, and glycerol have been used as calibration liquids (see also Table 1).

Apart from these liquids used for the calibration, measurement data for further samples are also shown. Note that, although a reasonable linear correlation can be observed, the uncalibrated raw data show significant deviations from the nominal values, which is mainly due to the thermal shunts not considered by the simple model mentioned above (see also [3],[4]). 


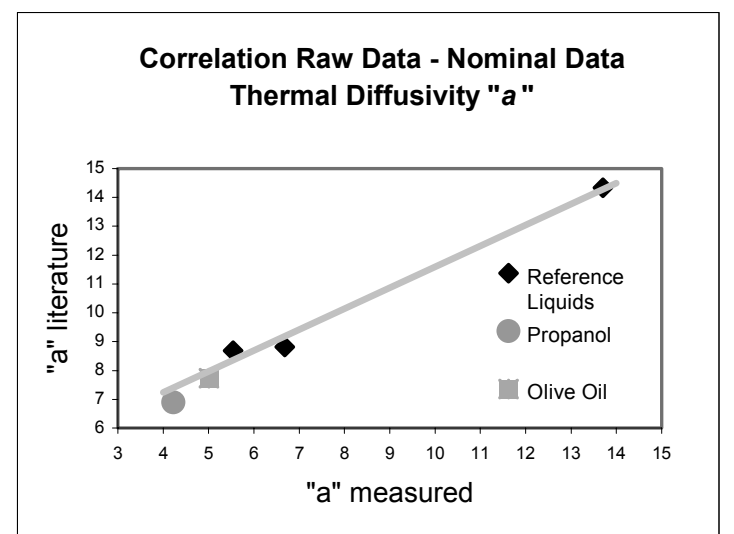

Fig. 4: Calibration diagram for the thermal diffusivity.

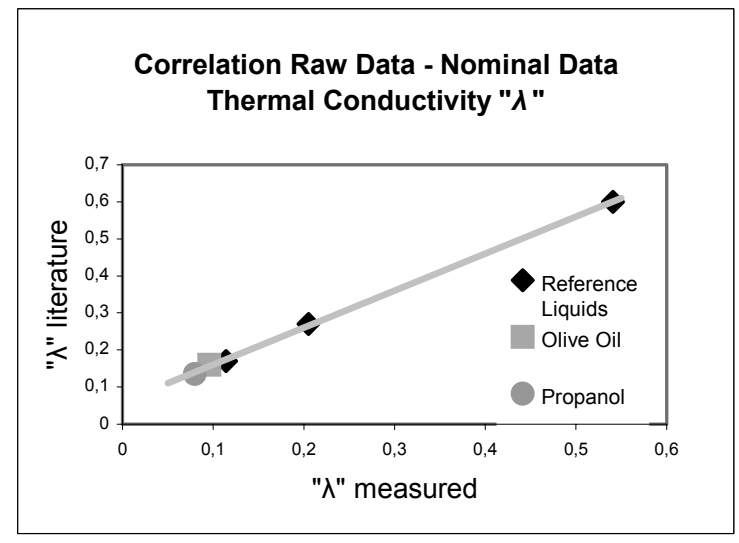

Fig. 5: Calibration diagram for the thermal conductivity.

\section{Results}

The read-out electronics were tested with five different liquids: Ethanol, Propanol, Water, Glycerol and Olive Oil, where the first three have been selected for the calibration procedure. Table one compares the measured results (after calibration) with the exact values found in the literature. Note that there is some deviation also for the data used in the linear fit partly due to the slight non-linearity in the correlation. Furthermore, after the calibration the liquids used for calibration have been measured again such that this deviation also includes reproducibility errors. The outcomes for the thermal conductivity are reasonably close to the exact values. A bigger difference occurs in the findings for the thermal diffusivity. For example the result for water is $16,3 \times 10^{-8} \mathrm{~m}^{2} / \mathrm{s}$ instead of $14,33 \times 10^{-8} \mathrm{~m}^{2} / \mathrm{s}, \mathrm{so}$ the relative error is 13,7 percent. This can partly be attributed to the accuracy of the nominal data for thermal material properties but also to the intrinsic errors of this prototype setup.

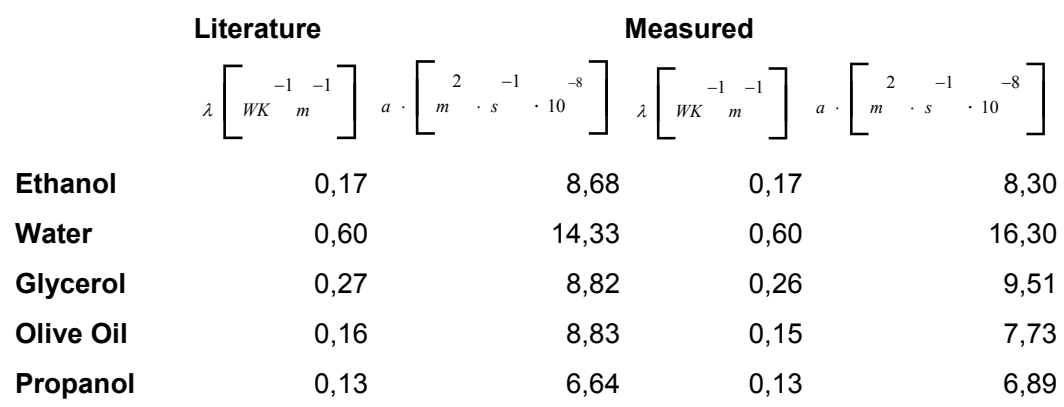

Table 1: Comparison of nominal data and measurement data (after calibration). The material data have been obtained from [5].

\section{Conclusions}

The presented read-out electronics show that the thermal properties of liquids can be measured with a compact and reasonably priced electronic circuit such that expensive laboratory equipment does not have to be used for this application. The accuracy of the circuit can be further improved, e.g., using AD/DA Converters with a higher resolution.

\section{References}

[1] J.J. Healy, J. J. de Groot, and J. Kesin: "The theory of the transient hot-wire method for measuring thermal conductivity", Physica C, vol. 82, no. 2, pp. 392-408, 1976.

[2] J. Kuntner, F. Kohl, B. Jakoby: Simultaneous thermal conductivity and diffusivity sensing in liquids using a micromachined device, Sensors and Actuators A 130-131 (2006): 62-67.

[3] J. Kuntner, A. Jachimowicz, F.Kohl, B. Jakoby: Determining Thermal Properties of Liquids: Membrane-based versus Bridge-based Micromachined Sensor, IEEE SENSORS 2006, EXCO, Daegu, Korea / October 22 25, 2006

[4] J. Kuntner, B. Jakoby, R. Beigelbeck, F. Kohl: Analysis of Spurious Effects in Membrane-Based Micromachined Thermal Conductivity Sensors.

[5] D.R. Lide, CRC Handbook of Chemistry and Physics, $84^{\text {th }}$ edition, CRC Press, LLC 2004. 